\title{
Position-force control of mobile manipulator - nonadaptive and adaptive case
}

\author{
MIRELA KACZMAREK, WOJCIECH DOMSKI and ALICJA MAZUR
}

\begin{abstract}
This article presents a control algorithm for nonholonomic mobile manipulators with a simple, geometric holonomic constraint imposed on the robot's arm. A mathematical model in generalized, auxiliary and linearized coordinates is presented, as well as the constrained dynamics of the robotic system. A position-force control law is proposed, both for the fully known robot's model, as well as for the model with parametric uncertainty in the dynamics. Theoretical considerations are supported by the results of computer simulations.
\end{abstract}

Key words: holonomic constraint, nonholonomic constraint, parametric uncertainty, adaptive control

\section{Introduction}

Mobile manipulators, which are built of a mobile platform and onboard manipulator, that is assumed to be a rigid one, increase their importance. This is caused by wide variety of tasks which they can perform, from manipulation and transportation in different areas (both home and industrial environment), through robots performing machining operations, to specialized rescue platforms [3]. Many of this applications require not only precise position control but also some kind of a force control [12]. Such situation occurs whenever a direct contact between the robot and the environment is necessary for a correct realization of a given task.

While modelling of mobile manipulators we have to consider different constraints appearing during the motion, which for platform and manipulator can be both - holonomic and nonholonomic. This gives us four possible configurations of mobile manipulators: type $(h, h)$ - both the platform and the manipulator holonomic, type $(h, n h)-\mathrm{a}$ holonomic platform with a nonholonomic manipulator, type $(n h, h)$ - a nonholonomic

The authors are with Department of Cybernetics and Robotics, Wrocław University of Science and Technology, ul. Janiszewskiego 11/17, 50-372 Wrocław, Poland.

E-mails: \{mirela.kaczmarek\}\{wojciech.domski\}\{alicja.mazur\}@ pwr.edu.pl

The work of Mirela Kaczmarek was supported by the Wrocław University of Science and Technology under the grant 0402/0192/16. The work of Wojciech Domski was supported by the Wrocław University of Science and Technology under the grant 0402/0103/16. The work of Alicja Mazur was supported by the Wrocław University of Science and Technology under the statutory grant 0401/0209/16.

Received 26.01.2017. 
platform with a holonomic manipulator, and finally type $(n h, n h)$ - both the platform and the manipulator nonholonomic. Undoubtedly, the most popular type is $(n h, h)$ which is considered in this article.

During the mobile manipulator modelling some uncertainty should be taken into the consideration. Manipulator can be armed with tools with different mass or can transport objects of an unknown inertia moment. To achieve a satisfying quality of the positionforce control, an adaptive version of control algorithm should be used.

This article presents a position-force control algorithm for a $(n h, h)$ mobile manipulator. A simple geometric holonomic constraint, defining a relationship among joint coordinates of manipulator, is considered. According to the approach of object's modeling, previously used in papers [4] and [11], the reduced dynamics will be presented. Presented algorithm is an extended version of works presented in [7] and [6]. Additionally to the previous works, its adaptive version is also considered.

\section{Model of the fully known mobile manipulator}

\subsection{Model in generalized coordinates}

A mobile manipulator consists of two subsystems: a mobile platform and a rigid manipulator. This robotic system can be described by vector of generalized coordinates $q=\left(q_{m}, q_{r}\right)^{T} \in \mathbb{R}^{n+p}$ and generalized velocities $\dot{q}=\left(\dot{q}_{m}, \dot{q}_{r}\right)^{T} \in \mathbb{R}^{n+p}$. Coordinates $q_{m} \in$ $\mathbb{R}^{n}$ represent the platform whereas $q_{r} \in \mathbb{R}^{p}$ describe the manipulator. Robot motion is determined by nonholonomic constraints defined by platform's mechanical structure and holonomic ones occurring in the manipulator.

The dynamics of manipulator mounted on the nonholonomic platform, after consideration of all constraints, can be presented as follows

$$
Q(q) \ddot{q}+C(q, \dot{q}) \dot{q}+D(q)=B(q) u+f_{n}+f_{h},
$$

where: $q \in \mathbb{R}^{n+p}$ - a vector of generalized coordinates, $Q(q)$ - a symmetrical, positive definite inertia matrix, $C(q, \dot{q})$ - a matrix of centripetal and Coriolis forces, $D(q) \in \mathbb{R}^{n+p}$ - a vector of gravity, $B(q)$ - an input matrix, $u$ - a vector of control signals, $f_{n}$ and $f_{h}$ - external forces arising from nonholonomic and holonomic constraints acting upon the system.

Knowing that $q=\left(q_{m}, q_{r}\right)^{T}$, equation (1) can be presented in a block form

$$
\left[\begin{array}{ll}
Q_{v} & Q_{v a} \\
Q_{a v} & Q_{a}
\end{array}\right]\left(\begin{array}{c}
\ddot{q}_{m} \\
\ddot{q}_{r}
\end{array}\right)+\left[\begin{array}{cc}
C_{v} & C_{v a} \\
C_{a v} & C_{a}
\end{array}\right]\left(\begin{array}{c}
\dot{q}_{m} \\
\dot{q}_{r}
\end{array}\right)+\left(\begin{array}{c}
0 \\
D_{a}
\end{array}\right)=\left[\begin{array}{cc}
B & 0 \\
0 & I
\end{array}\right]\left(\begin{array}{c}
u_{m} \\
u_{r}
\end{array}\right)+f_{n}+f_{h} .
$$

From this point, we assume that the motion of the mobile platform will be restricted by nonholonomic constraints, whereas the manipulator has holonomic constraints, resulting from its structure.

The platform should move without slippage of wheels that implicates existence of $l$ nonholonomic constraints presented in Pfaffian form 


$$
A\left(q_{m}\right) \dot{q}_{m}=0
$$

These constraints act on the system through forces $f_{n}$ which should always fulfil equation (3). According to d'Alembert principle [5], forces resulting from constraints, can be expressed as

$$
f_{n}^{T} d q_{m}=0 .
$$

From (3) and (4) it can be concluded that force coming from nonholonomic constraints depends on nonholonomic Lagrange multipliers as follows

$$
f_{n}=\left(\begin{array}{c}
A^{T}\left(q_{m}\right) \lambda_{n} \\
0
\end{array}\right),
$$

where nonholonomic Lagrange multipliers $\lambda_{n} \in \mathbb{R}^{l}$ represent static friction forces preventing platform wheels slippage.

Holonomic forces, acting on manipulator joints, come from $k$ geometric holonomic constraints

$$
\phi\left(q_{r}\right)=0 .
$$

Such forces have following form

$$
f_{h}=\left(\begin{array}{c}
0 \\
J^{T} \lambda_{h}
\end{array}\right),
$$

where $J$ is Jacobi matrix of holonomic constraints

$$
J=\frac{\partial \phi}{\partial q}=\left[\begin{array}{ll}
\frac{\partial \phi}{\partial q_{m}} & \frac{\partial \phi}{\partial q_{r}}
\end{array}\right]=\left[\begin{array}{ll}
0 & J_{r}
\end{array}\right]
$$

and $\lambda_{h}$ denotes a vector of forces occurring between manipulator's end-effector and surfaces defined by holonomic constraint. Using virtual work concept, it is not necessary to measure real forces acting on surface with end-effector because they can be equivalently measured in manipulator joints as the $f_{h}$ vector [12]. The force transformation from the robot workspace to the joint workspace is performed by using the transposed Jacobi matrix.

\subsection{Model in auxiliary velocities}

From (3) we can clearly see that allowed platform velocities belong to kernel of Pfaffian matrix: $\dot{q}_{m} \in \operatorname{Ker} A\left(q_{m}\right)$. Therefore, nonholonomic constraints can be expressed as follows

$$
\dot{q}_{m}=G\left(q_{m}\right) \eta,
$$

where $\eta$ constitutes a vector of auxiliary velocities [1], $G$ is an orthogonal complement matrix which fulfils $A\left(q_{m}\right) G\left(q_{m}\right)=0$. It allows elimination of Lagrange multipliers in 
the platform's dynamics equations by left-sided multiplication by $G^{T}$ matrix, $G^{T} A^{T}=0$ condition is preserved. After this step, dynamics equations (2) have a form

$$
\begin{gathered}
{\left[\begin{array}{cc}
G^{T} Q_{v} G & G^{T} Q_{v a} \\
Q_{a v} G & Q_{a}
\end{array}\right]\left(\begin{array}{c}
\dot{\eta} \\
\ddot{q}_{r}
\end{array}\right)+\left[\begin{array}{cc}
G^{T}\left(C_{v} G+Q_{v} \dot{G}\right) & G^{T} C_{v a} \\
C_{a v} \dot{G}+Q_{a v} G & C_{a}
\end{array}\right]\left(\begin{array}{c}
\eta \\
\dot{q}_{r}
\end{array}\right)} \\
+\left(\begin{array}{c}
0 \\
D_{a}
\end{array}\right)=\left[\begin{array}{cc}
G^{T} B & 0 \\
0 & I
\end{array}\right]\left(\begin{array}{c}
u_{m} \\
u_{r}
\end{array}\right)+\left(\begin{array}{c}
0 \\
J_{r}^{T} \lambda_{h}
\end{array}\right) .
\end{gathered}
$$

\subsection{Model in linearizing coordinates}

The coordinates $q_{m}$ related to mobile platform can be partially linearized [1]. For this purpose we introduce a local coordinate transformation [2]

$$
\xi=\left(\begin{array}{l}
\xi_{1} \\
\xi_{2}
\end{array}\right)=\Phi\left(q_{m}\right)=\left(\begin{array}{c}
h\left(q_{m}\right) \in \mathbb{R}^{m} \\
k\left(q_{m}\right) \in \mathbb{R}^{n-m}
\end{array}\right),
$$

where $\xi_{1}=h\left(q_{m}\right) \in \mathbb{R}^{m}$ denotes a vector of linearizing coordinates, $k\left(q_{m}\right) \in \mathbb{R}^{(n-m)}$ are variables chosen in such a way that $\Phi\left(q_{m}\right)$ transformation is a local diffeomorphism. Variables $k\left(q_{m}\right)$ are non-linearized coordinates of the platform. Linearized variables depend on the auxiliary velocities in the following way

$$
\dot{\xi}_{1}=\frac{\partial h}{\partial q_{m}} \dot{q}_{m}=\frac{\partial h}{\partial q_{m}} G \eta=R^{-1}\left(q_{m}\right) \eta .
$$

The selection of linearizing variables is not trivial - they must be chosen to fulfil condition $\operatorname{det} R^{-1}\left(q_{m}\right) \neq 0$.

Model of the manipulator's dynamics along with the mobile platform, defined in linearized coordinates, becomes following

$$
M_{1} \ddot{w}+C_{1} \dot{w}+D_{1}=B_{1} u+f_{h},
$$

with elements defined as below

$$
\begin{gathered}
M_{1}=\left[\begin{array}{cc}
R^{T} G^{T} Q_{v} G R & R^{T} G^{T} Q_{v a} \\
Q_{a v} G R & Q_{a}
\end{array}\right], \\
C_{1}=\left[\begin{array}{cc}
R^{T} G^{T}\left[C_{v} G R+Q_{v}(\dot{G} R+G \dot{R})\right] & R^{T} G^{T} C_{v a} \\
C_{a v} G R+Q_{a v}(G \dot{R}+\dot{G} R) & C_{a}
\end{array}\right], \\
D_{1}=\left(\begin{array}{c}
0 \\
D_{a}
\end{array}\right), B_{1}=\left[\begin{array}{cc}
R^{T} G^{T} B & 0 \\
0 & I
\end{array}\right], \\
u=\left(\begin{array}{c}
u_{m} \\
u_{r}
\end{array}\right), f_{h}=\left(\begin{array}{c}
0 \\
J_{r}^{T} \lambda_{h}
\end{array}\right), w=\left(\begin{array}{l}
\xi_{1} \\
q_{r}
\end{array}\right) .
\end{gathered}
$$




\subsection{Constrained dynamics}

Configuration space of the manipulator with holonomic constraints (i.e. geometric constraints) is reduced to $(p-k)$ degrees of freedom. Vector of manipulator's coordinates $q_{r}$ may be presented as $q_{r}=\left(q_{1 r}, q_{2 r}\right)$, where $q_{1 r} \in \mathbb{R}^{p-k}$ contains coordinates, that are constrained, while $q_{2 r} \in \mathbb{R}^{k}$ denotes rest of the coordinates. Jacobi matrix $J_{\xi}$, recalculated for linearized platform coordinates, can be obtained by differentiating constraint equation (6) by coordinates of the platform and the manipulator

$$
J_{\xi}=\left[\frac{\partial \phi}{\partial \xi_{1}}, \frac{\partial \phi}{\partial q_{1 r}}, \frac{\partial \phi}{\partial q_{2 r}}\right]=\left[0,0, \frac{\partial \phi}{\partial q_{2 r}}\right]
$$

Based on [8], coordinates $q$ may be described as functions of $\zeta=\left(\xi_{1}, q_{1 r}\right)^{T}$ where

$$
w=\left(\begin{array}{l}
\xi_{1} \\
q_{r}
\end{array}\right)=\varphi(\zeta) .
$$

After differentiating, equation takes following form

$$
\dot{w}=\left(\begin{array}{c}
\dot{\xi}_{1} \\
\dot{q}_{r}
\end{array}\right)=\frac{\partial \varphi}{\partial \zeta} \dot{\zeta}=L(\zeta) \dot{\zeta}
$$

while following condition is fulfilled

$$
L(\zeta)^{T} J_{\xi}^{T}=0
$$

Using relationship (18) for (13) we receive a model of the robot's dynamics reduced to a surface of holonomic constraint. It has following form

$$
M_{1} L(\zeta) \ddot{\zeta}+C_{1} \dot{\zeta}+D_{1}=B_{1} u+J_{\xi}^{T} \lambda_{h}
$$

Left-sided multiplication of (19) by $L^{T}$ eliminates Lagrange multipliers $\lambda_{h}$ due to relationship (18). Therefore, the equation transforms to

$$
L^{T}(\zeta) M_{1} L(\zeta) \ddot{\zeta}+L^{T}(\zeta) C_{1} \dot{\zeta}+L^{T}(\zeta) D_{1}=L^{T}(\zeta) B_{1} u
$$

Assuming that

- $M_{L}=L^{T}(\zeta) M_{1} L(\zeta)$,

- $C_{L}=L^{T}(\zeta) C_{1}$,

- $D_{L}=L^{T}(\zeta) D_{1}$, 
equation (20) can be rewritten as follows

$$
M_{L} \ddot{\zeta}+C_{L} \dot{\zeta}+D_{L}=L^{T}(\zeta) B_{1} u
$$

The holonomic Lagrange multipliers $\lambda_{h}$ can be calculated by double differentiation, in time domain, the holonomic constraints equation (6)

$$
\ddot{\phi}\left(q_{r}\right)=\dot{J}_{\xi} \dot{w}+J_{\xi} \ddot{w}=0 .
$$

After substituting $\ddot{w}$ from dynamics equation of the mobile manipulator (13), the equation (22) takes a form

$$
\ddot{\phi}\left(q_{r}\right)=J_{\xi} M_{1}^{-1} J_{\xi}^{T} \lambda_{h}+\dot{J}_{\xi} \dot{w}-J_{\xi} M_{1}^{-1}\left(C_{1} \dot{w}+D_{1}-B_{1} u\right)=W(\phi) .
$$

Equation (22) does not guarantee that $\phi\left(q_{r}\right)=0$ will be always fulfilled. To assure it, even if manipulator is in some distance from surface of holonomic constraint, a numerical damping terms [9] are added to the system in the form of following equation

$$
W(\phi)=\ddot{\phi}\left(q_{r}\right)+2 \alpha \dot{\phi}\left(q_{r}\right)+\beta^{2} \phi\left(q_{r}\right),
$$

where $\alpha$ and $\beta$ coefficients should guarantee asymptotic stability.

After the above steps, the holonomic Lagrange multipliers fulfil

$$
J_{\xi} M_{1}^{-1} J_{\xi}^{T} \lambda_{h}=W(\phi)-\dot{J}_{\xi} \dot{w}+J_{\xi} M_{1}^{-1}\left(C_{1} \dot{w}+D_{1}-B_{1} u\right) .
$$

For constrained dynamics we assume that holonomic constraints are fulfilled. In such a case it holds $\dot{J}_{\xi}=0$ and $W(\phi)=0$ (because manipulator is located in holonomic constraint, not near it). The holonomic Lagrange multipliers can be calculated as follows

$$
\lambda_{h}=\left(J_{\xi} M_{1}^{-1} J_{\xi}\right)^{-1} J_{\xi} M_{1}^{-1}\left(C_{1} \dot{w}+D_{1}-B_{1} u\right)=Z\left(C_{1} \dot{w}+D_{1}-B_{1} u\right),
$$

where $Z$ is well defined for independent holonomic constraints.

\section{Control law for nonadaptive case}

In case of full knowledge about mobile manipulator's dynamics, control law consists of the two parts: a position controller and a force controller.

Control input for the system is defined as follows

$$
B_{1} u=B_{1} u_{p}-J_{\xi}^{T} u_{f}
$$

where $u_{p}$ denotes the position control law for constrained dynamics and $u_{f}$ denotes the force control law. For constrained coordinates values of $u_{p}$ are always equal to 0 .

Considered system is constrained in nonholonomic (3) and holonomic (6) way that makes coordinates $\xi_{1}, q_{1 r}$ and $q_{2 r}$ not independent. Coordinate $q_{2 r}$, which is constrained, 
is unambiguously defined by $\left(\xi_{1}, q_{1 r}\right)$ coordinates, therefore there is no need to control it.

In this article, some version of Sadegh \& Horowitz control algorithm [10] has been chosen as the position control law

$$
L^{T} B_{1} u_{p}=M_{L}(q) \ddot{\zeta}_{r}+C_{L}(q, \dot{q}) \dot{\zeta}_{r}+D_{L}(q)-K_{d} s-K_{p} e
$$

where $e=\zeta-\zeta_{d}-$ a position error, $s=\dot{\zeta}-\dot{\zeta}_{r}=\dot{e}+\Lambda e-$ a sliding variable, $\zeta_{r}-$ a reference (artificial) trajectory, $K_{p}=K_{p}^{T}>0, K_{d}=K_{d}^{T}>0, \Lambda=\Lambda^{T}>0$ - parameters of the controllers.

The force control is defined as follows

$$
u_{f}=Z M_{1}\left(\ddot{w}_{d}-K_{1} \dot{e}_{w}-K_{0} e_{w}\right)+\lambda_{h d}-K_{f} e_{\lambda}
$$

where $K_{0}, K_{1}, K_{f}>0$ are regulation parameters and $e_{w}=w-w_{d}$ is the position tracking error.

Proof of the position errors convergence Consider the Lyapunov function as below

$$
V(s, e)=\frac{1}{2} s^{T} M_{L} s+\frac{1}{2} e^{T} K_{p} e
$$

which is nonnegative due to the positive definiteness of $M_{L}$ and $K_{p}$. For such the function $V$ the following evaluation holds

$$
\begin{aligned}
V(s, e) & \leqslant \frac{1}{2} \bar{\lambda}_{q}\left(M_{L}\right)\|s\|^{2}+\frac{1}{2} \bar{\lambda}\left(K_{p}\right)\|e\|^{2} \\
& \leqslant \alpha\left(\|s\|^{2}+\|e\|^{2}\right)
\end{aligned}
$$

where $\bar{\lambda}_{q}\left(M_{L}\right)$ denotes maximal eigenvalue of $M_{L}$ matrix estimated over all values $q$, $\bar{\lambda}\left(K_{p}\right)$ is maximal eigenvalue of regulation matrix $K_{p}$, while

$$
\alpha=\max \left(\frac{1}{2} \bar{\lambda}_{q}\left(M_{L}\right), \frac{1}{2} \bar{\lambda}\left(K_{p}\right)\right)>0 .
$$

Equations of system (21) with closed loop of feedback control law (25) are equal to

$$
M_{L} \dot{s}+C_{L} s+K_{d} s+K_{p} e=0 .
$$

Calculating the time derivative of $\mathrm{V}$ given by (27) along trajectories of the closed-loop system (29) and evoking a skew-symmetry, between inertia matrix $M_{L}$ and matrix of Coriolis and centrifugal forces $C_{L}$, we obtain following expression 


$$
\begin{aligned}
\dot{V} & =\frac{1}{2} s^{T} \dot{M}_{L} s+s^{T} M_{L} \dot{s}+e^{T} K_{p} \dot{e} \\
& =\frac{1}{2} s^{T} \dot{M}_{L} s+s^{T}\left(-C_{L} s-K_{d} s-K_{p} e\right)+e^{T} K_{p} \dot{e} \\
& =-s^{T} K_{d} s-s^{T} K_{p} e+e^{T} K_{p}(s-\Lambda e) \\
& =-s^{T} K_{d} s-e^{T} K_{p} \Lambda e \\
& \leqslant-\beta\left(\|s\|^{2}+\|e\|^{2}\right) \quad \beta=\min \left(\underline{\lambda}\left(K_{d}\right), \underline{\lambda}\left(K_{p} \Lambda\right)\right)>0 \\
& \leqslant 0,
\end{aligned}
$$

where $\underline{\lambda}(\cdot)$ denotes minimal eigenvalue of a matrix.

Transforming (28) and (30) we get

$$
\dot{V} \leqslant-\beta\left(\|s\|^{2}+\|e\|^{2}\right) \leqslant-\frac{\beta}{\alpha} V \quad \longrightarrow \quad \dot{V}+\frac{\beta}{\alpha} V \leqslant 0 .
$$

After multiplying both sides of the equation (31) by $\exp \left\{\frac{\beta}{\alpha} t\right\}$ and integrating in time, we obtain following expression

$$
V(t) e^{\frac{\beta}{\alpha} t}-V(0) \leqslant 0 \quad \longrightarrow \quad V(t) \leqslant V(0) e^{-\frac{\beta}{\alpha} t} .
$$

Using the inequality (32), the exponentially fast convergence of tracking errors $e$ and $s$ can be proved

$$
\begin{gathered}
\frac{1}{2} \underline{\lambda}_{q}\left(M_{L}\right)\|s\|^{2} \leqslant \frac{1}{2} s^{T} M_{L} s \leqslant V(t) \leqslant V(0) e^{-\frac{\beta}{\alpha} t}, \\
\frac{1}{2} \underline{\lambda}\left(K_{p}\right)\|e\|^{2} \leqslant \frac{1}{2} e^{T} K_{p} e \leqslant V(t) \leqslant V(0) e^{-\frac{\beta}{\alpha} t},
\end{gathered}
$$

and, consequently

$$
\begin{aligned}
& \|s\| \leqslant \sqrt{\frac{2 V(0)}{\underline{\lambda}_{q}\left(M_{L}\right)}} \cdot e^{-\frac{\beta}{2 \alpha} t}, \\
& \|e\| \leqslant \sqrt{\frac{2 V(0)}{\underline{\lambda}\left(K_{p}\right)}} \cdot e^{-\frac{\beta}{2 \alpha} t} .
\end{aligned}
$$

It ends the proof of exponentially fast convergence of position errors to 0 .

Convergence of a force error The equation (23), describing real force acting on holonomic surface, can be rewritten as below

$$
\lambda_{h}=-Z M_{1} \ddot{w}+u_{f} .
$$

Next, force error fulfills following equation

$$
\lambda_{h}=-Z M_{1} \ddot{w}+Z M_{1}\left(\ddot{w}_{d}-K_{1} \dot{e}_{w}-K_{0} e_{w}\right)+\lambda_{h d}-K_{f} e_{\lambda},
$$


where $K_{1}, K_{0}, K_{f}>0$ are regulation parameters and $e_{\lambda}=\lambda_{h}-\lambda_{h d}$ is a force error.

The equation (33) can be transformed into a form

$$
\left(I+K_{f}\right) e_{\lambda}=-Z M_{1}\left(\ddot{e}_{w}+K_{1} \dot{e}_{w}+K_{0} e_{w}\right) .
$$

It can be seen, that the force error depends on the position tracking error and its derivatives. It is easy to show that the right side of (34) is bounded and, therefore, the force error $e_{\lambda}$ can be made arbitrarily small by the choice of large value of $K_{f}$.

\section{Control law for adaptive case}

It has been considered so far, how to control fully known mobile manipulator. Now we want to present, how to control mobile manipulator when the full model of such object is unknown. In such a case there are two possibilities - the structural uncertainty, when forms of some matrices in dynamical model are unknown, and the parametric uncertainty, when we do not know certain number of model parameters. In this article, the second type is being considered and therefore adaptive version of Sadegh \& Horowitz algorithm is proposed.

The nonadaptive position control law (25) can be transformed to modified version

$$
L^{T} B_{1} u_{p}=M_{O L}(q) \ddot{\zeta}_{r}+C_{O L}(q, \dot{q}) \dot{\zeta}_{r}+D_{O L}(q)+Y\left(q, \dot{q}, \dot{\zeta}_{r}, \ddot{\zeta}_{r}\right) a-K_{d} s-K_{p} e,
$$

where $M_{O L}, C_{O L}, D_{O L}$ represent known parts of model, and $Y a$ is unknown one. $Y$ is so-called regression matrix and $a$ is a vector of unknown coefficients of the robot model.

If parameters $a$ are unknown, then we can use their time estimates denoted as $\hat{a}(t)$. These estimates are calculated from so-called adaptation law as follows

$$
\dot{\hat{a}}(t)=\dot{\widetilde{a}}(t)=-\Gamma Y^{T}\left(q, \dot{q}, \dot{\zeta}_{r}, \ddot{\zeta}_{r}\right) s,
$$

where $\Gamma=\Gamma^{T}>0$ is a matrix of adaptive gains and $\widetilde{a}=\hat{a}-a$ is a vector of parameter errors.

Proof of the convergence of position errors Consider the following Lyapunov function

$$
V_{a}(s, e)=\frac{1}{2} s^{T} M_{L} s+\frac{1}{2} e^{T} K_{p} e+\frac{1}{2} \widetilde{a}^{T} \Gamma^{-1} \widetilde{a} .
$$

Equations of system (21) with closed loop of feedback control law (35) are equal to

$$
M_{L} \dot{s}+C_{L} s+K_{d} s+K_{p} e-Y\left(q, \dot{q}, \dot{\zeta}_{r}, \ddot{\zeta}_{r}\right) \widetilde{a}(t)=0 .
$$

Calculating the time derivative of $V_{a}$ defined by (37) along trajectories of the closed-loop system (36)-(38) and evoking a skew-symmetry between the inertia matrix $M_{L}$ and the matrix of Coriolis and centrifugal forces $C_{L}$, we obtain following expression 


$$
\begin{aligned}
\dot{V}_{a} & =\frac{1}{2} s^{T} \dot{M}_{L} s+s^{T} M_{L} \dot{s}+e^{T} K_{p} \dot{e}+\widetilde{a}^{T} \Gamma^{-1} \dot{\tilde{a}} \\
& =\frac{1}{2} s^{T} \dot{M}_{L} s+s^{T}\left(-C_{L} s-K_{d} s-K_{p} e+Y\left(q, \dot{q}, \dot{\zeta}_{r}, \ddot{\zeta}_{r}\right) \widetilde{a}\right)+e^{T} K_{p} \dot{e}+\widetilde{a}^{T} \Gamma^{-1} \dot{\widetilde{a}} \\
& =-s^{T} K_{d} s-s^{T} K_{p} e+s^{T} Y\left(q, \dot{q}, \dot{\zeta}_{r}, \ddot{\zeta}_{r}\right) \widetilde{a}+e^{T} K_{p}(s-\Lambda e)-\widetilde{a}^{T} Y^{T}\left(q, \dot{q}, \dot{\zeta}_{r}, \ddot{\zeta}_{r}\right) s \\
& =-s^{T} K_{d} s-e^{T} K_{p} \Lambda e \\
& \leqslant 0 .
\end{aligned}
$$

From La Salle Invariance Principle it can be concluded that $(s, e)=(0,0)$ is asymptotically stable equilibrium point of the system (38). Convergence of the force error to arbitrarily small value can be proved using similar arguments as for the nonadaptive case. It ends the proof.

\section{Simulations}

The simulations have been done using the MATLAB package and the SIMULINK toolbox. As an object of simulations we have taken RR manipulator mounted on a unicycle, presented in Fig. 1.

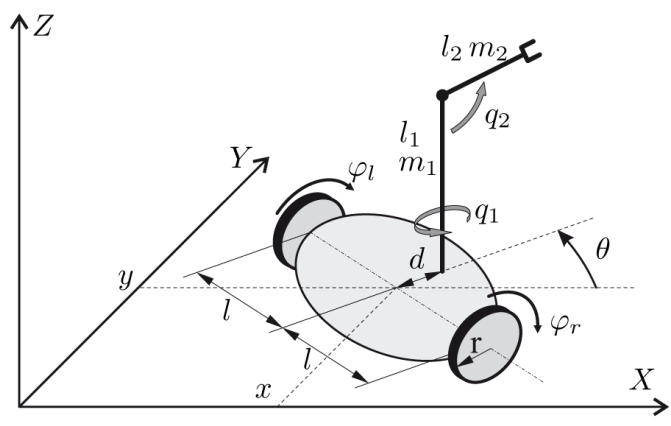

Figure 1: Schematic of modelled mobile manipulator

Links of the RR manipulator have been modeled as homogenous sticks with length equal to $l_{1}=0.9 \mathrm{~m}, l_{2}=1 \mathrm{~m}$ and masses $m_{1}=m_{2}=20 \mathrm{~kg}$. Parameters of platform were equal to: $m_{c}=50 \mathrm{~kg}, l=0.3 \mathrm{~m}, r=1.5 \mathrm{~m}, d=0.1 \mathrm{~m}$.

\subsection{Model in generalized coordinates}

For the mobile manipulator presented in Fig. 1, the following generalized robot coordinates have been chosen

$$
q=\left(\begin{array}{c}
q_{m} \\
q_{r}
\end{array}\right)
$$


where $q_{m}=\left(x, y, \theta, \phi_{l}, \phi_{r}\right)^{T}$, and $q_{r}=\left(q_{1}, q_{2}\right)$. Dynamics of mobile manipulator is given by the equation (2) with elements equal to

- $Q$ - symmetric positive definite inertia matrix

$$
Q=\left[\begin{array}{ccccc|cc}
Q_{11} & 0 & Q_{13} & 0 & 0 & Q_{16} & Q_{17} \\
0 & Q_{22} & Q_{23} & 0 & 0 & Q_{26} & Q_{27} \\
Q_{31} & Q_{32} & Q_{33} & 0 & 0 & Q_{36} & Q_{37} \\
0 & 0 & 0 & 0 & 0 & 0 & 0 \\
0 & 0 & 0 & 0 & 0 & 0 & 0 \\
\hline Q_{61} & Q_{62} & Q_{63} & 0 & 0 & Q_{66} & 0 \\
Q_{71} & Q_{72} & Q_{73} & 0 & 0 & 0 & Q_{77}
\end{array}\right]=\left[\begin{array}{cc}
Q_{v} & Q_{v a} \\
Q_{a v} & Q_{a}
\end{array}\right]
$$

where elements are defined below

$$
\begin{aligned}
Q_{11} & =m_{1}+m_{2}, \\
Q_{13} & =Q_{31}=-a s_{01} c_{2}-\left(m_{1}+m_{2}\right) d s_{0}, \\
Q_{16} & =Q_{61}=-a s_{01} c_{2}, \\
Q_{17} & =Q_{71}=-a c_{01} s_{2}, \\
Q_{22} & =m_{1}+m_{2}, \\
Q_{23} & =Q_{32}=a c_{01}+\left(m_{1}+m_{2}\right) d c_{0}, \\
Q_{26} & =Q_{62}=a c_{01} c_{2}, \\
Q_{27} & =Q_{72}=-a s_{01} s_{2}, \\
Q_{33} & =\frac{1}{2} m_{1} l_{1} d s_{0} c_{01}+b c_{2}^{2}+m_{2} l_{2} d c_{1} c_{2}+m_{2} d^{2}, \\
Q_{36} & =Q_{63}=b c_{2}^{2}+a d c_{1} c_{2}, \\
Q_{37} & =Q_{73}=-a d s_{1} s_{2}, \\
Q_{66} & =b c_{2}^{2}, \\
Q_{77} & =b,
\end{aligned}
$$

and symbols denote

$$
\begin{gathered}
c_{0}=\cos \theta, s_{0}=\sin \theta, c_{1}=\cos q_{1}, s_{1}=\sin q_{1}, c_{2}=\cos q_{2}, s_{2}=\sin q_{2}, \\
c_{01}=\cos \left(\theta+q_{1}\right), s_{01}=\sin \left(\theta+q_{1}\right), a=\frac{m_{2} l_{2}}{2}, b=\frac{m_{2} l_{2}^{2}}{3} .
\end{gathered}
$$


- $C$ - matrix of centripetal and Coriolis forces

$$
C=\left[\begin{array}{ccccc|cc}
0 & 0 & C_{13} & 0 & 0 & C_{16} & C_{17} \\
0 & 0 & C_{23} & 0 & 0 & C_{26} & C_{27} \\
0 & 0 & C_{33} & 0 & 0 & C_{36} & C_{37} \\
0 & 0 & 0 & 0 & 0 & 0 & 0 \\
0 & 0 & 0 & 0 & 0 & 0 & 0 \\
\hline 0 & 0 & C_{63} & 0 & 0 & C_{66} & C_{67} \\
0 & 0 & C_{73} & 0 & 0 & C_{76} & 0
\end{array}\right]=\left[\begin{array}{cc}
C_{v} & C_{v a} \\
C_{a v} & C_{a}
\end{array}\right]
$$

with elements equal to

$$
\begin{aligned}
& C_{13}=-\left(\left(m_{1}+m_{2}\right) d c_{0}+a c_{01} c_{2}\right) \dot{\theta}-a c_{01} c_{2} \dot{q}_{1}+a s_{01} s_{2} \dot{q}_{2} \\
& C_{16}=-a c_{01} c_{2} \dot{\theta}-a c_{01} c_{2} \dot{q}_{1}+a s_{01} s_{2} \dot{q}_{2} \\
& C_{17}=a s_{01} s_{2} \dot{\theta}+a s_{01} s_{2} \dot{q}_{1}-a c_{01} c_{2} \dot{q}_{2} \\
& C_{23}=\left(-\left(m_{1}+m_{2}\right) d s_{0}-a s_{01} c_{2}\right) \dot{\theta}-a s_{01} c_{2} \dot{q}_{1}-c_{01} s_{2} \dot{q}_{2} \\
& C_{26}=-a s_{01} c_{2} \dot{\theta}-a s_{01} c_{2} \dot{q}_{1}-a c_{01} s_{2} \dot{q}_{2} \\
& C_{27}=-a c_{01} s_{2} \dot{\theta}-a c_{01} s_{2} \dot{q}_{1}-a s_{01} c_{2} \dot{q}_{2} \\
& C_{33}=\frac{m_{1} l_{1}}{4} d\left(c_{01} c_{0}-s_{01} s_{0}\right) \dot{\theta}-\left(a d c_{2} s_{1}+\frac{m_{1} l_{1}}{4} d s_{0} s_{01}\right) \dot{q}_{1}-\left(a d c_{1} s_{2}+b c_{2} s_{2}\right) \dot{q}_{2}, \\
& C_{36}=-\left(a d s_{1} c_{2}+\frac{m_{1} l_{1}}{4} d s_{01} s_{0}\right) \dot{\theta}-a d c_{2} s_{1} \dot{q}_{1}-\left(a d c_{1}+b c_{2}\right) s_{2} \dot{q}_{2}, \\
& C_{37}=-\left(a d c_{1}+b c_{2}\right) s_{2} \dot{\theta}-\left(a d c_{1}+b c_{2}\right) s_{2} \dot{q}_{1}-a d c_{2} s_{1} \dot{q}_{2} \\
& C_{63}=\left(a d c_{2} s_{1}+\frac{m_{1} l_{1}}{4} s_{0} s_{01}\right) \dot{\theta}-b s_{2} c_{2} \dot{q}_{2} \\
& C_{66}=-b s_{2} c_{2} \dot{q}_{2} \\
& C_{67}=-b s_{2} c_{2} \dot{\theta}-b s_{2} c_{2} \dot{q}_{1} \\
& C_{73}=\left(a d s_{2} c_{1}+b c_{2} s_{2}\right) \dot{\theta}+b c_{2} s_{2} \dot{q}_{1} \\
& C_{76}=b s_{2} c_{2} \dot{\theta}+b s_{2} c_{2} \dot{q}_{1} .
\end{aligned}
$$

- $D$ - vector of gravity

$$
D=\left(\begin{array}{c}
0 \\
-m_{2} \frac{l_{2}}{2} g c_{2}
\end{array}\right) .
$$

\subsection{Constraints}

The considered mobile platform is entirely nonholonomic. It means that there is no longitudinal and lateral slipping of both wheels. These conditions can be written in the 
following form:

$$
\left\{\begin{array}{l}
\dot{x} \sin \theta-\dot{y} \cos \theta=0, \\
\dot{x} \cos \theta+\dot{y} \sin \theta-l \dot{\theta}-r \dot{\phi}_{l}=0, \\
\dot{x} \cos \theta+\dot{y} \sin \theta+l \dot{\theta}-r \dot{\phi}_{r}=0 .
\end{array}\right.
$$

These constrains may be presented in Pfaffian form (3) with following $A$ matrix

$$
A=\left[\begin{array}{ccccc}
\sin \theta & -\cos \theta & 0 & 0 & 0 \\
\cos \theta & \sin \theta & -l & -r & 0 \\
\cos \theta & \sin \theta & l & 0 & -r
\end{array}\right]
$$

In turn, the manipulator is restricted with holonomic constraint

$$
\Phi=l_{1}+l_{2} \sin q_{2}=0 .
$$

\subsection{Model in linearizing coordinates}

The linearized coordinates of the mobile platform can be selected as follows

$$
\xi_{1}=\left(\begin{array}{c}
x+e \cos \theta \\
y+e \sin \theta
\end{array}\right),
$$

where $e$ is distance between "guidance point", which coordinates have been chosen as linearizing outputs, from the centre of mass [2]. Matrices $G\left(q_{m}\right), R\left(q_{m}\right)$ from (14) equation can be defined as follows

$$
\begin{aligned}
G\left(q_{m}\right)= & {\left[\begin{array}{cc}
\cos \theta & \cos \theta \\
\sin \theta & \sin \theta \\
\frac{1}{L} & -\frac{1}{L} \\
0 & \frac{2}{R} \\
\frac{2}{R} & 0
\end{array}\right], } \\
R\left(q_{m}\right)= & {\left[\begin{array}{cc}
\cos \theta & \sin \theta \\
-\frac{1}{e} \sin \theta & \frac{1}{e} \cos \theta
\end{array}\right], \quad \operatorname{det} R\left(q_{m}\right)=\frac{1}{e} \neq 0 . }
\end{aligned}
$$

The vector of linearized coordinates is equal to

$$
\left(\begin{array}{c}
\xi_{1} \\
q_{r}
\end{array}\right)=\left(\begin{array}{c}
x+e \cos \theta \\
y+e \sin \theta \\
q_{1} \\
q_{2}
\end{array}\right)
$$


Jacobi matrix, expressed in coordinates (47), has a form

$$
J_{\zeta}=\left[\frac{\partial \phi}{\partial \xi_{1}}, \frac{\partial \phi}{\partial q_{r}}\right]=\left[\begin{array}{llll}
0 & 0 & 0 & l_{2} \cos q_{2}
\end{array}\right]
$$

Coordinates, which are not holonomically constrained, have been chosen as

$$
\zeta=\left(\begin{array}{c}
x+e \cos \theta \\
y+e \sin \theta \\
q_{1}
\end{array}\right)
$$

For these coordinates the desired trajectory has been defined as below

$$
\zeta_{d}=\left(\begin{array}{c}
1.5 \sin t \\
1.5 \cos t \\
\frac{\pi}{4}(1-\cos t)
\end{array}\right) .
$$

Desired force value is $\lambda_{d}=10 \mathrm{~N}$.

\subsection{Results}

During the simulation, the following control parameters have been chosen: $K_{p}=\operatorname{diag}\{1000\}, K_{d}=\operatorname{diag}\{100\}, \Lambda=\operatorname{diag}\{10\}, K_{f}=100, K_{0}=\operatorname{diag}\{100\}$ and $K_{1}=\operatorname{diag}\{10\}$. In adaptive case we have used the same parameters and also $\Gamma=1000$.

During simulations we have assumed, that the parameter $b=\frac{m_{2} l_{2}^{2}}{3}$, defining the inertia moment of the payload, is unknown. It responses the situation, that the shape of the transported payload is unknown.

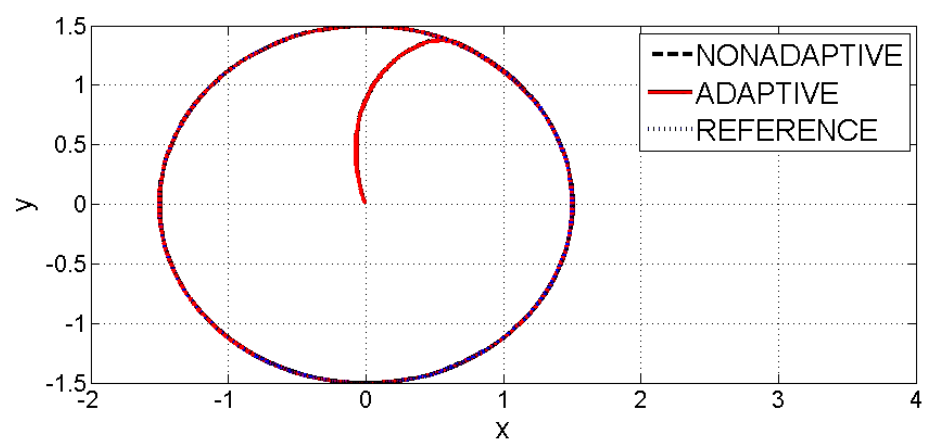

Figure 2: Trajectory tracking for mobile platform.

In Fig. 2 tracking of the desired trajectory by the mobile platform for nonadaptive and adaptive case has been presented. During the simulation study it was observed, that 
the time required for the mobile platform to reach the desired trajectory, was dependent on control parameters and initial conditions. The differences between the motion of the platform in the nonadaptive and the adaptive case are small, what is natural, because the estimated parameter is related to the manipulator, not to the platform.

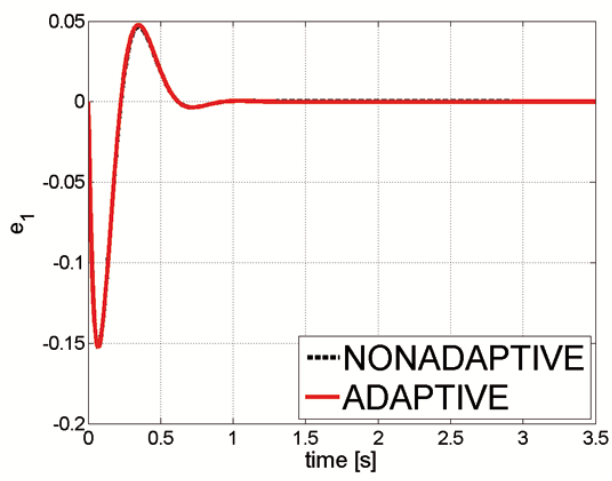

(a) Error $e_{1}=\zeta_{1}-\zeta_{1 d}$

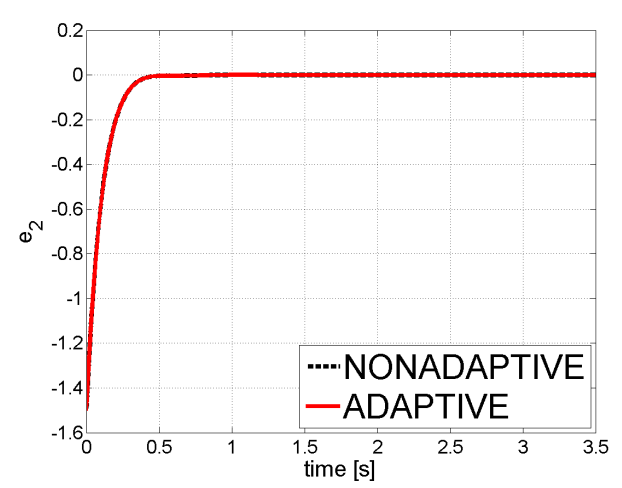

(b) Error $e_{2}=\zeta_{2}-\zeta_{2 d}$

Figure 3: Tracking errors for the platform

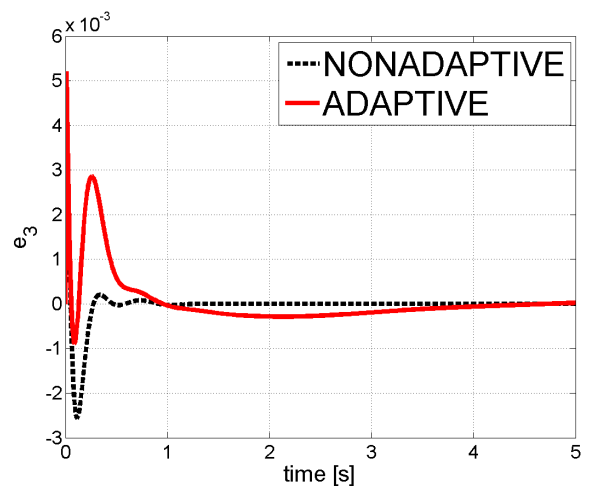

(a) Error $e_{3}=\zeta_{3}-\zeta_{3 d}$

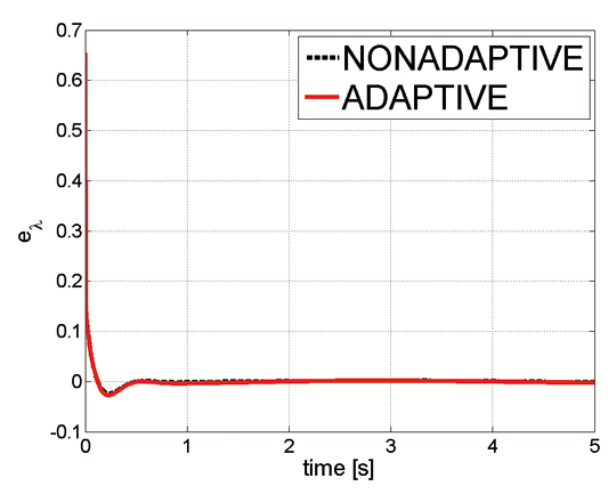

(b) Error $e_{\lambda}=\lambda_{h}-\lambda_{h d}$

Figure 4: Tracking errors for unconstrained manipulator variables and force

Plots presented in Figs. 3 and 4 show the trajectory tracking process, position errors and force error in time domain. Position errors of the platform: $e_{1}=\zeta_{1}-\zeta_{1 d}$ and $e_{2}=$ $\zeta_{2}-\zeta_{2 d}$; along with $e_{3}=q_{1}-q_{1 d}$ (the error of joint position $q_{1}$ of the manipulator) all converge to 0 . The time, after which the errors converge, and control quality in terms of overshooting, is dependent on regulation parameters $K_{p}$ and $K_{d}$. In turn, the force error $e_{\lambda}=\lambda_{h}-\lambda_{h d}$, as expected, is limited and it has steady-state error close to 0 (it is only practically stable). Its level depends on $K_{f}$ control parameter. 


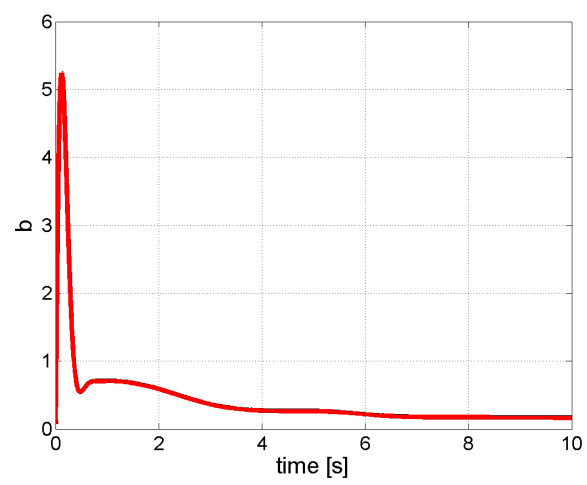

Figure 5: Parameter estimation in time.

In the adaptive case, for error $e_{1}$ and $e_{2}$, which are related to platform, there is only a small difference in comparison to nonadaptive case. For $e_{3}$ we can observe slower convergence - it means, that adaptive version needs more time to converge to 0 . It is obvious and it results from theory: proofs have shown that the nonadaptive control law guarantees exponentially fast convergence to 0 and the adaptive case is only asymptotically convergent to 0 .

Selection of the different control algorithm, in comparison to [7], had almost none effect. However, the position control algorithm has been changed while the force control algorithm has been extended.

It can be observed that the obtained force tracking error, in initial phase, is one order higher in magnitude in comparison to force error presented in [7], however, it converges to the same level.

\section{Conclusions}

In this article the control algorithm of nonholonomic mobile manipulator with geometric holonomic constraint has been presented. The control law consists of two stages - the positional and the force part. The position control algorithm has been applied to the variables without holonomic constraints. The variable with geometrical holonomic constraint has been controlled using the force control algorithm.

Presented algorithm is a modification of the work presented in [4] and is an extension of the work presented in [7]. In this paper also a methodology of obtaining reduced dynamics for a $(n h, h)$ class of mobile manipulator has been described.

In further research we intend to apply different holonomic constraints such as static obstacles as well as geometric constraints, dependant on all state variables. 


\section{References}

[1] G. Campion, G. Bastin and B. D'Andrea Novel: Structural properties and classification of kinematic and dynamic models of wheeled mobile robots. IEEE Trans. on Robotics and Automation, 12(5), (1996).

[2] C. Canudas de Wit, B. Siciliano and G. Bastin: Theory of Robot Control. Springer, London, 1996.

[3] H. Früh, P. Keller and T. Perucchi: Intelligent Mobile Manipulators in Industrial Applications: Experiences and Challenges, pages 370-385. Springer, Berlin, 2007.

[4] Z. LI, S. S. GE and A. MING: Adaptive robust motion/force control of holonomicconstrained nonholonomic mobile manipulators. IEEE Trans. on Systems, Man and Cybernetics - part B: Cybernetics, 37(3), (2007), 607-616.

[5] A. MAZUR: Model-based control for nonholonomic mobile manipulators. Printing House of Wrocław University of Technology, Wrocław, 2009, (in Polish).

[6] A. Mazur, M. KaczmareK and W. Domski: Position and force control of mobile manipulator, pages 257-262, (in Polish). Printing House of Warsaw University of Technology, Warsaw, 2015.

[7] A. Mazur, M. Kaczmarek and W. Domski: Position-force control of nonholonomic mobile manipulator with simple holonomic constraint. In 10th Int. Workshop on Robot Motion and Control, Poznań, Poland, (2015), 257-262.

[8] N. H. McClamroch and D. Wang: Feedback stabilization and tracking of constrained robots. IEEE Trans. on Automatic Control, 33(5), (1988), 419-426.

[9] J. K. Mills and A. A. GoldenberG: Force and position control of manipulators during constrained motion tasks. IEEE Trans. on Robotics and Automation, 5(1), (1989), 30-46.

[10] N. SADEgh and R. Horowitz: Stability and robustness analysis of a class of adaptive controllers for robotic manipulators. Int. J. of Robotics Research, 9(3), (1990), 74-94.

[11] C. SU and Y. STEPANENKO: Robust motion/force control of mechanical systems with classical nonholonomic constraints. IEEE Trans. on Automatic Control, 39(3), (1994), 609-614.

[12] L. Villani and J. De Schutter: Force control. In B. Siciliano and O. Khatib, editors, Springer Handbook of Robotics, Springer, 2008, 161-185. 